Revista Ingeniería y Región. 2016;15(1): 23-35

\title{
Desarrollo de un modelo para la planificación integral del recurso hídrico en la cuenca hidrográfica del Río Aipe, Huila, Colombia
}

\section{Development of a model for integral planning of water resources in Aipe catchment, Huila, Colombia}

\author{
Andrés F. Labrador C. ${ }^{1}$, Juan M. Zúñiga L. ${ }^{2}$ y Jonathan Romero C. ${ }^{3}$
}

\begin{abstract}
Resumen
El propósito del estudio fue desarrollar un modelo hidrológico para conocer el impacto de los escenarios de cambio climático sobre la oferta hídrica para el período (2011-2050), como herramienta para apoyar la toma de decisiones en la planificación integral del recurso hídrico en la cuenca hidrográfica del Río Aipe, Huila. Colombia. La cuenca del río Aipe $\left(688.9 \mathrm{Km}^{2}\right)$ fue seleccionada como área de estudio representativa de cuencas semiáridas de clima tropical con problemas de gestión de recursos hídricos. El modelo WEAP (Water Evaluation and Planning System) fue calibrado y validado comparado el caudal simulado y observado en el punto de cierre de la cuenca (estación puente carretera). Los resultados muestran la habilidad del modelo para simular el comportamiento hidrológico de la cuenca a escala diaria $\left(\mathrm{R}^{2}=0.75\right)$. Respecto a la simulación de los escenarios, la línea base (1980-2011) estimó un caudal medio de $15.31 \mathrm{~m}^{3} \mathrm{~s}^{-1}$, el escenario I estimó un caudal de $14.88 \mathrm{~m}^{3} \mathrm{~s}^{-1}(-2.81 \%)$, el escenario II de $10.87 \mathrm{~m}^{3} \mathrm{~s}^{-1}(-29 \%)$, escenario III de $18.98 \mathrm{~m}^{3} \mathrm{~s}$ ${ }^{1}(+23.97 \%)$ y escenario IV de $5.24 \mathrm{~m}^{3} \mathrm{~s}^{-1}(-65.77 \%)$. Esto podría ocasionar numerosos problemas económicos y conflictos sociales, en términos de desabastecimiento de agua potable y disminución de volúmenes de riego para producción de cultivos agropecuarios. El modelo hidrológico desarrollado representa una herramienta útil que asiste el proceso de planeamiento hidrológico a partir de escenarios multicriterios que tienen en cuenta los múltiples y opuestos usos del recurso hídrico. Además, el modelo contribuye a mejorar el sistema de gestión integral del recurso hídrico y apoyar la toma de decisiones, a través de conocimiento confiable de cómo responden las cuencas en términos hidrológico ante distintos escenarios climáticos y de demanda hídrica.
\end{abstract}

Palabras clave: WEAP, cambio climático, hidrología tropical, modelación hidrológica, gestión integral de recursos hídricos.

1 Ingeniero Agrícola. Universidad Surcolombiana - Neiva. Av. Pastrana - Carrera 1. felipe_9009@hotmail.com

2 Ingeniero Agrícola. Universidad Surcolombiana - Neiva. Av. Pastrana - Carrera 1. juan21591@hotmail.com

3 MSc Hidrosistemas. Universidad Surcolombiana/CENIGAA - Neiva. Av. Pastrana - Carrera 1. jonathan.romero. cuellar@gmail.com

Recibido: 20 noviembre 2015. Aprobado: 15 febrero 2016. 


\begin{abstract}
The purpose of this study is to develop a hydrological model to understand the impact of climate change scenarios on the water supply for the period, 2011-2050. The Aipe catchment (area $688.9 \mathrm{Km}^{2}$ ) in Huila, Colombia was selected for this study. The WEAP model (water evaluation and planning system) was calibrated and validated for the stream flows of the Aipe catchment. The results showed the ability of the model to simulate the daily stream flow $\left(\mathrm{R}^{2}=0.75\right)$. From the simulated scenarios, the baseline $(1980-2011)$ estimated an average flow of $15.31 \mathrm{~m}^{3} \mathrm{~s}^{-1}$; scenario I estimated a rate $14.88 \mathrm{~m}^{3} \mathrm{~s}^{-1}(-2.81 \%)$; scenario II 10.87 $\mathrm{m}^{3} \mathrm{~s}^{-1}(-29 \%)$; scenario III $18.98 \mathrm{~m}^{3} \mathrm{~s}^{-1}(+23.97 \%)$ and scenario IV $5.24 \mathrm{~m}^{3} \mathrm{~s}^{-1}(-65.77 \%)$. These flow rates could result in numerous economic problems and social conflicts in terms of drinking water shortages and a decrease in irrigation volumes for agricultural production. The hydrological model developed for the Aipe river catchment is a useful tool to assists the hydrological planning process; its multiple scenarios take into account the numerous, competing uses for water resources. In addition, the WEAP model helps improve the system of integrated management of water resources, and supports decision-making process through reliable knowledge of how to respond to catchments in hydrologic terms considering various climate scenarios and water demand.
\end{abstract}

Keywords: WEAP, climate change, tropical hydrology, hydrology modeling, integrated water resources management

\section{Introducción}

La planificación integral del recurso hídrico es un proceso participativo que pretende garantizar un uso sostenible del agua a escala de cuenca (Arrojo, et al., 2015). Entendiendo el uso sostenible del agua como aquel que mantiene la multifuncionalidad del recurso hídrico (Martínez y Esteve, 2004). Sin embargo, el concepto de multifuncionalidad puede llegar a ser confuso. Para efectos de este artículo y desde la gobernanza del agua se pueden identificar tres funciones básicas: (i) el agua como fuente de vida (derecho humano), asociado a la sostenibilidad de los ecosistemas, caudales ecológicos, calidad del agua y equidad intergeneracional. (ii) agua - ciudadanía (servicios públicos) muy asociado al abastecimiento de agua potable, tratamiento de aguas residuales y en general saneamiento básico. (iii) agua - productividad, asociado a las funciones productivas que generan beneficios económicos (Baños, et al. 2014). Por otro lado, Suárez et al. (2014) propone que la finalidad de la planificación integral del recurso hídrico es mejorar la eficiencia del uso del agua (razón económica), fomentar la equidad del acceso al agua (razón social) y garantizar la sostenibilidad (razón ambiental).

Además, de las múltiples interpretaciones asociadas al concepto de sostenibilidad y funcionalidad del agua, la planificación integral del recurso hídrico envuelve un al to grado de complejidad (Baños, et al., 2016). Las causas de esta complejidad se pueden resumirse en: (i) megadiversidad de la naturaleza con procesos ecohidrológicos altamente sensibles a la interacción con actividades productivas. (ii) enfoque transdiciplinario, la gestión del agua implica la participación de enfoques: jurídicos, ecológicos, hidrológicos, económicos, territoriales, agronómicos, sociológicos, políticos, espirituales y culturales. (iii) integración de subsistemas naturales (cuencas hidrográficas, ríos, acuíferos, lagos, humedales, costas, etc.) y artificiales (acueductos, sistemas de riego, represas, plantas de tratamiento de aguas residuales, infraestructura hidráulica, etc.) (iv) diversidad de actores sociales y ámbito de aplicación de la planificación del recurso hídrico; en este ámbito interactúa conocimiento científico, procedimientos técnicos, gestores sociales, modelos de desarrollo, ciudadanos, espacios de gobernanza, toma de decisiones, etc. Toda esta realidad simplificada en pocas palabras hace de la planificación integral del recurso hídrico un desafio del siglo XXI.

Dentro de las alternativas más utilizadas para afrontar el desafio de la planificación integral del recurso hídrico se encuentra la modelación hidrológica participativa (Sivapalan, et al., 2014; Castro, et al., 2014). La modelación hidrológica de cuencas hidrográficas se plantea como una herramienta para apoyar la toma de decisiones entorno a la gestión del agua. La modelación hidrológica suministra varias ventajas para la gestión del agua: (i) mejora el conocimiento del proceso a evaluar; (ii) sistematiza e incrementa la información ambiental de la cuenca (Ruiz-Pérez, et al., 2016); (iii) simula predicciones a corto, mediano y largo plazo; (iv) evalúa escenarios como alternativas de gestión; 
(v) estima la incertidumbre y el riesgo de la toma de decisiones. Todas estas ventajas pretender suministrar elementos de juicio para tomar la mejor decisión. Especialmente a través de la exploración de las consecuencias de distintas condiciones y alternativas de gestión (escenarios de cambio climático, uso del suelo, demanda hídrica, etc.), la integración e interacción de los diferentes elementos de la cuenca, la gestión participativay la producción de indicadores ambientales (Baños, et al., 2016).

Recientemente uno de los principales problemas que más preocupa a la planificación integral del recurso hídrico es el impacto del cambio climático sobre la oferta hídrica (Ruiz-Villanueva, et al., 2015). A nivel global existe un consenso entre diferentes combinaciones de escenarios de emisiones, modelos de ciclos biogeoquímicos y modelos climáticos en donde el IPCC estima que la temperatura global en el año 2100 será entre $1.4^{\circ} \mathrm{C}$ y $5.8^{\circ} \mathrm{C}$ más alta. Sin embargo, el calentamiento global no será uniforme. A su vez, se supone que existirá mayor alternancia y extremos en la distribución de los inviernos y las sequias. A nivel regional las proyecciones de cambio climático para el Huila muestran un incremento de $2^{\circ} \mathrm{C}$ en la temperatura media para el año 2040, que puede ser aún mayor si se parte de las nuevas proyecciones mundiales, así como una disminución de hasta el $30 \%$ en la precipitación (Ruiz, 2010). Pese a la alta incertidumbre que presentan las proyecciones de cambio climático Thompson et al. (2013) es necesario tenerlas en cuenta como escenarios posibles del futuro para diseñar una serie de medidas que minimicen el impacto del cambio climático. En ese sentido, se pueden planificar mejor la inversión de recursos, evitando temas de reconstrucción, reasentamiento y restauración.

Teniendo en cuenta la potencia de los modelos hidrológicos en planificación hídrica, la comunidad científica ha desarrollado gran cantidad de herramientas. En este sentido y de acuerdo con Beven (2001) seleccionar un modelo hidrológico no es una tarea fácil. Sin embargo, hay algunas recomendaciones al momento de seleccionar un modelo hidrológico: (i) el objetivo de la modelación o el propósito del modelo (¿se ajusta a nuestro problema?); (ii) ¿las entradas del modelo se pueden adquirir en el tiempo y el presupuesto del proyecto?; (iii) ¿las hipótesis del modelo son asumibles para el caso de estudio? (condiciones de escala espacial y temporal); (iv) existe un modelo robusto disponible y vale la pena invertir tiempo y dinero; (v) existe suficiente documentación y soporte de acceso libre (Martínez-Fernández, et al., 2013).
En el caso de estudio de la cuenca del río Aipe se abordó una problemática de gestión de recursos hídricos con planificación a largo plazo incluyendo las proyecciones de los escenarios de cambio. Además la cuenca tenía un estudio previo de modelación hidrológica con modelos agregados tipo tanques (Thornthwaite y abcd Thomas) (Romero, et al., 2015). En este sentido, se buscaba un modelo semi-distribuido (para contrastar el desempeño de los modelos), con un componente fuerte en la gestión de las demandas hídricas, un módulo de gestión de embalse y con la posibilidad de incorporar los diferentes puntos de vista de los actores de la cuenca de forma participativa. Acorde con las razones expuestas el modelo que se seleccionó fue WEAP(Water Evaluation and Planning System).

El modelo weap cuenta con muchas implementaciones en diferentes partes del mundo, como California, Massachusetts, Georgia, Sur y Norte de África, varios países asiáticos (Xue, et al., 2015). Específicamente en la región Andina se encuentran aplicaciones en Chile. Por ejemplo, se implementó en cuencas Nivales para simular los caudales futuros de acuerdo con las proyecciones de cambio climático para los períodos 2036-2065 y 2071-2100 (Mardones, 2009). De igual forma, en la cuenca Andina del rio Teno (Chile) se utilizó el modelo weap para evaluar la vulnerabilidad del sector de los recursos hídricos, con el fin de contribuir al proceso de toma de decisiones sobre medidas de adaptación frente al cambio climático (Mena, 2009). Por otro lado, en Ecuador, en la cuenca del rio Pastaza, se utilizó el modelo weap para conocer el comportamiento hidrológico de la cuenca (Lema y Plaza, 2009).

De acuerdo con la problemática planteada y los antecedentes, el objetivo del trabajo consistió en desarrollar un modelo hidrológico para conocer el impacto de los escenarios de cambio climático sobre la oferta hídrica para el período (2011 - 2050), como herramienta para apoyar la toma de decisiones en la planificación integral del recurso hídrico en la cuenca hidrográfica del Río Aipe, Huila, Colombia.

\section{Materiales y métodos}

\subsection{Localización y descripción de la cuenca}

La Cuenca hidrográfica del río Aipe está localizada en el noroccidente del departamento del Huila, sur de Colombia. Se localiza con las coordenadas $3^{\circ} 13^{\prime} \mathrm{N} \mathrm{y}$ $75^{\circ} 14^{\prime} \mathrm{O}$, cubre un área de $688.9 \mathrm{Km}^{2}$ y se extiende 
entre los municipios de Aipe, Neiva y Palermo. La longitud del cauce principal es $51.31 \mathrm{~km}$. El río Aipe es tributario del río Magdalena. De acuerdo con sus características morfométricas es una cuenca de tamaño grande, con densidad de drenaje muy bajo $\left(0.0732 \mathrm{~km}^{-1}\right)$, lo que indica un drenaje pobre y un predominio de los procesos de ladera dando origen a mayores tiempos de residencia ( 10.53 horas) y menores caudales picos. Este supuesto se confirma con el coeficiente de compacidad (2.29) el cual indica una cuenca con forma oval oblonga poco propensa a crecientes súbitas. El rango altitudinal varía entre $390-1900 \mathrm{msnm}$. El clima de la cuenca se distribuye acorde con el gradiente altitudinal entre húmedo, seco y muy seco. La precipitación media mensual oscila entre 100 y $170 \mathrm{~mm}$. La temperatura media mensual es de $27.57^{\circ} \mathrm{C}$ con humedad relativa de $70.75 \%$.

La cuenca del río Aipe juega un papel fundamental para el desarrollo del municipio de Aipe. El río Aipe abastece el acueducto principal del municipio y ocho acueductos veredales, de igual forma abastece la extracción de hidrocarburos, la irrigación de los cultivos de arroz (riego por superficie), la producción piscícola y ganadería de leche y carne. En resumen, del río Aipe dependen las principales actividades productivas del municipio y como se evidencia son sectores productivos que demandan baste recurso hídrico. Esta situación ha generado un conflicto por el uso del agua, básicamente porque las demandas se incrementan y la oferta hídrica es la misma, sino disminuye por fenómenos de variabilidad y cambio climático.

\subsection{Descripción del modelo Weap}

WEAP (Water Evaluation and Planning System) es una iniciativa del Stockholm Environment Institute. Esta herramienta computacional tiene un modelo hidrológico conceptual semi-distribuido acoplado con un sistema integrado de planeación de recursos hídricos que permite apoyar la toma de decisiones. Weap se apoya en escenarios multicriterios para el planeamiento hidrológico de cuencas hidrográficas incorporando acueductos, sistemas de riego, producción hidroeléctrica y múltiples estrategias de manejo del recurso hídrico. La herramienta incorpora subrutinas para representar procesos como el cálculo de la oferta y demanda hídrica, escorrentía, infiltración, requerimientos hídricos para cultivos, flujos y almacenamiento de agua, generación, tratamiento, descarga de contaminantes y de calidad de agua en ríos para variados escenarios hidrológicos y de políticas. Para los escenarios se evalúa una amplia gama de opciones de manejo del agua y tiene en cuenta los múltiples y opuestos usos de los recursos hídricos. Para todo esto cuenta con una interfaz gráfica basada en mapas SIG con resultados flexibles como mapas, gráficos y tablas (Sieber y Purkey, 2007). Específicamente este trabajo se enfocó en la utilización del módulo de hidrología a través del método de humedad del suelo, que simula el proceso lluvia - escorrentía en unidades de respuesta hidrológicas (URH) por medio de dos tanques, los cuales pretenden estimar los flujos superficiales y subterráneos. Este método unidimensional se fundamenta en funciones empíricas que describen la evapotranspiración, escorrentía superficial, flujo subsuperficial y percolación profunda (Duque y Vázquez, 2015).

\subsection{Datos y esquema de modelación}

A nivel climático la cuenca del río Aipe esta monitoreada por 12 estaciones pluviométricas y dos estaciones climatológicas ordinarias (San Alfonso y Santa María) las cuales tienen registros diarios desde 1970 - 2011 (figura 1). Además, en el punto de cierre de la cuenca se utilizaron los registros de caudales diarios desde 1980 - 2011 (tabla 1). Todas las estaciones hidrometeorológicas utilizadas son administradas por el Instituto de Hidrología, Meteorología y Estudios Ambientales de Colombia (IDEAM). La información de la precipitación fue regionalizada mediante el proceso de Polígonos de Thiessen. Adicionalmente, las series de precipitación y temperatura media fueron afectadas por un factor de corrección acorde con el gradiente altitudinal de la cuenca hidrográfica.

La Figura 1 muestra el esquema de modelación que se realizó para la cuenca del río Aipe. La cuenca fue modelada con weap a escala de subcuencas y estas, a su vez, por bandas de elevación cada 500 metros, configurando así un total de 65 unidades de respuesta hidrológicas o catchments. Se modelaron en total 20 corrientes tributarias directas e indirectas del cauce principal con la información generada en 1 punto de monitoreo de caudal denominado estación Puente Carretera (2113703). Se consideraron, además, 3 demandas de agua para consumo, los cuales son los centros poblados de San Francisco, Los Órganos y el acueducto municipal de Aipe, utilizando la ecuación del método Geométrico del capítulo B del (RAS - 2000), también los requerimientos hídricos para las principales coberturas vegetales (bosques, pastos, suelos desnudos, zonas agrícolas, zonas urbanas, lagos, café, aguas abiertas, arroz, sorgo y cacao). 
Tabla 1. Estaciones hidrometeorológicas con influencia en la cuenca del Río Aipe

\begin{tabular}{|c|c|c|c|}
\hline Nombre & Tipo* & Coordenadas & $\begin{array}{c}\text { Elevación } \\
\text { (msnm) }\end{array}$ \\
\hline El Carmen & PM & $3^{\circ} 0^{\prime} 25.7^{\prime \prime} \mathrm{N} 75^{\circ} 33^{\prime} 33.1^{\prime \prime} \mathrm{W}$ & 2250 \\
\hline Casa de Zinc & PM & $3^{\circ} 17^{\prime} 19.4^{\prime \prime} \mathrm{N} 75^{\circ} 35^{\prime} 26.9^{\prime \prime} \mathrm{W}$ & 1700 \\
\hline Gaitana & PM & $3^{\circ} 5^{\prime} 41.1^{\prime \prime} \mathrm{N} 75^{\circ} 40^{\prime} 38.6^{\prime \prime} \mathrm{W}$ & 1500 \\
\hline La Julia & PM & $3^{\circ} 5^{\prime} 55.6^{\prime \prime} \mathrm{N} 75^{\circ} 31^{\prime} 48.1^{\prime \prime} \mathrm{W}$ & 1691 \\
\hline Órganos & PM & $3^{\circ} 7^{\prime} 15^{\prime \prime} \mathrm{N} 75^{\circ} 31^{\prime} 48.1^{\prime \prime} \mathrm{W}$ & 800 \\
\hline Hacienda Pérez & PM & $3^{\circ} 14^{\prime} 36.8^{\prime \prime} \mathrm{N} 75^{\circ} 15^{\prime} 21.4^{\prime \prime} \mathrm{W}$ & 450 \\
\hline Potrerito & PM & $3^{\circ} 8^{\prime} 27.9^{\prime \prime} \mathrm{N} 75^{\circ} 22^{\prime} 46.1^{\prime \prime} \mathrm{W}$ & 850 \\
\hline Praga & PM & $3^{\circ} 16^{\prime} 6.9^{\prime \prime} \mathrm{N} 75^{\circ} 29^{\prime} 26.4^{\prime \prime} \mathrm{W}$ & 1085 \\
\hline Rioclaro & PM & $3^{\circ} 8^{\prime} 8^{\prime \prime} \mathrm{N} 75^{\circ} 39^{\prime} 3.8^{\prime \prime} \mathrm{W}$ & 2230 \\
\hline San Luis & PM & $3^{\circ} 4^{\prime} 50.3^{\prime \prime} \mathrm{N} 75^{\circ} 29^{\prime} 4.8^{\prime \prime} \mathrm{W}$ & 1140 \\
\hline San Pedro & PM & $3^{\circ} 15^{\prime} 0^{\prime \prime} \mathrm{N} 75^{\circ} 27^{\prime} 0^{\prime \prime} \mathrm{W}$ & 850 \\
\hline La Yeguera & PM & $3^{\circ} 18^{\prime} 19.2^{\prime \prime} \mathrm{N} 75^{\circ} 12^{\prime} 2.8^{\prime \prime} \mathrm{W}$ & 400 \\
\hline Pte. Carretera & LG & $3^{\circ} 15^{\prime} 48.3^{\prime \prime} \mathrm{N} 75^{\circ} 15^{\prime} 3.49^{\prime \prime} \mathrm{W}$ & 385 \\
\hline
\end{tabular}

*Tipo de estación: PM: Pluviométrica, LG: Limnigráfica.

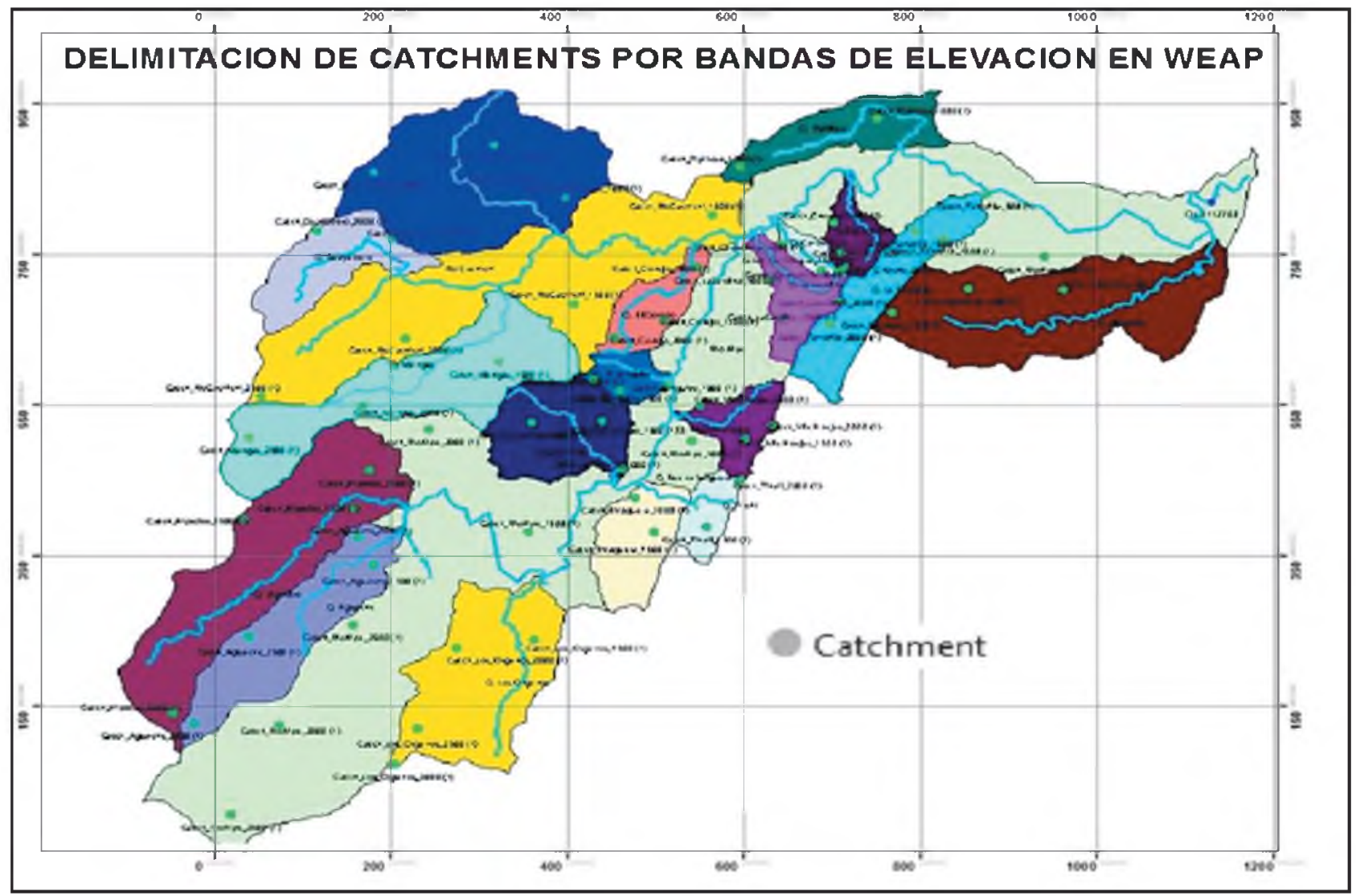

Figura 1. Esquema de modelación con las unidades de respuesta hidrológica de la cuenca del río Aipe. 


\subsection{Calibración, validación y evaluación del modelo}

La calibración y validación del modelo fue desarrollada utilizando los caudales observados en el punto de cierre de la cuenca (estación Puente Carretera). Durante la fase de calibración se utilizó el $70 \%$ de la serie histórica de caudales observados (1980-2001). La calibración se realizó a través de conocimiento experto en hidrología y el método de prueba y error. Este método implica un ajuste manual de parámetros basado en el criterio del investigador, conociendo la dinámica del modelo, la calidad de la información ambiental y la escala de los procesos naturales de la cuenca en estudio. Una vez calibrado el modelo se procedió a la validación del mismo con el 30\% de los datos de caudales observados restantes (2002 - 2011). Tanto la calibración como la validación fueron evaluadas con la métrica del coeficiente de determinación $\left(\mathrm{R}^{2}\right)$, cuyo principal objetivo es establecer una correlación confiable entre los valores simulados y observados. El coeficiente determina la calidad del modelo para replicar los resultados, y la proporción de variación de los resultados, que pueden explicarse por el modelo.

$$
R^{2}=1-\frac{\sum\left(Q_{s}-Q_{o b s}\right)^{2}}{\sum\left(Q_{o b s}-\overline{Q_{o b s}}\right)^{2}}
$$

Donde,

$Q_{o b s}=$ Promedio del caudal observado;

$Q_{o b s}=$ Caudal observado; $Q_{s}=$ Caudal simulado.

\subsection{Escenarios de población y cambio climático}

La primera hipótesis plantea la creación de un escenario base de referencia para el cálculo de la oferta hídrica asociado a un incremento poblacional. Actualmente y de acuerdo con los registro del municipio la población de referencia es 23.513 habitantes. Luego esa población de referencia se incrementar un $2 \%$ a partir de una proyección geométrica (escenario I, 23.983 habitantes) y luego se incrementa un 10\% (escenario II, 25.864 habitantes), estos incrementos de la población tiene un impacto directo sobre la demanda y la oferta hídrica los cuales serán representados por el modelo hidrológico.

La segunda hipótesis hace referencia a un cambio del clima. Esta hipótesis es más complicada dada la incertidumbre que manejan los escenarios de cambio climático, la variedad de modelos climáticos y las posibilidades de los escenarios. En este sentido, se uti- lizó una herramienta basada en múltiples modelos y escenarios, para ilustrar el rango de los cambios posibles en el clima durante el siglo 21 (Angarita, 2014). El propósito de la metodología es generar series de proyecciones climáticas de la variable de precipitación para la cuenca del río Aipe, a nivel de las unidades de análisis del modelo WEAP, informadas por las predicciones del proyecto CMIP5 (Coupled Model Intercomparison Project Phase 5), que sumadas al clima actual, permitan establecer un rango de comportamiento hidrológico posible en la cuenca del río Aipe para los años 2010 a 2050. Finalmente del ensamble de posibilidades se selecciona una tendencia climática húmeda (escenario III) y una climática seca (escenario IV) para evaluar su respuesta hidrológica (tabla 2).

Tabla 2. Resumen de los escenarios para la cuenca del río Aipe.

\begin{tabular}{lc}
\hline Nombre & Variable \\
\hline Escenario I & $+2 \%$ población \\
Escenario II & $+10 \%$ población \\
Escenario III & Clima húmedo \\
Escenario IV & Clima seco \\
\hline
\end{tabular}

\section{Resultados y discusión}

\subsection{Calibración del modelo}

El modelo weap se corrió a nivel diario con unos valores iniciales de los parámetros acorde con la inferencia de la información ambiental (mapa de suelos, cobertura vegetal, balance hídrico, mapa geológico etc.). Luego se realizó un análisis de sensibilidad preliminar para identificar los parámetros más sensibles. Los parámetros más sensibles están en función del tipo de respuesta hidrológica. Por ejemplo, se identificó que los caudales máximos se afectan primordialmente por el factor de resistencia a la escorrentía (RRF), la conductividad hidráulica en la zona de raíces (Ks) y la capacidad de retención de humedad en la capa superior del suelo (SWC). Cuando el valor de SWC se incrementa se logra un mayor flujo subsuperficial, y por lo tanto el aumento de caudales máximos. Por otro lado, el flujo base es muy sensible a los parámetros: dirección preferencial de flujo (PFD) y la conductixvidad hidráulica en la zona profunda (Kd). Al aumentar el valor de PFD aumenta la conductividad en la zona profunda, $y$, por lo tanto, la descarga de perfiles profundos, y como consecuencia aumenta los caudales bases. De igual forma, 
regulado la transmisión del flujo base se podrá ajustar la capacidad de retención de humedad en las capas profundas del suelo (DWC). En resumen los parámetros que mejor se ajustaron en el modelo hidrológico fueron: el factor de resistencia a la escorrentía (RRF), la conductividad hidráulica en la zona de raíces (RZC), la conductividad hidráulica en la zona profunda (DC), dirección preferencial de flujo (PFD), la capacidad de retención de humedad en la capa superior (SWC) y en la capa profunda (DWC). La tabla 3 muestra los rangos de los parámetros para cada una de las coberturas utilizadas, debido a que es un modelo semi-distribuido no hay un único valor de parámetros óptimos, por el contrario cada unidad de respuesta hidrológica tiene su conjunto de parámetros óptimos.

Luego de la identificación de los parámetros más sensibles y su rango de acción sobre el modelo se procedió a realizar la calibración manual del modelo; comparando el caudal simulado y observado en el punto de cierre de la cuenca (estación puente carretera). Algunos autores como Gupta, et al., (1998) y Francés, et al., (2007) recomiendan la calibración automática a través de una función objetivo y algoritmos de optimización como los de Monte Carlo o genéticos etc. Estos autores consideran que la calibración manual es tediosa y requiere de un hidrólogo con bastante experiencia para que el modelo se pueda calibrar, lo cual no es siempre posible en los proyectos. En contraste, Beven y Ferrer (2001) difieren de la calibración auto- mática porque las funciones objetivos tienen alta dimensionalidad, múltiples óptimos y superficies complejas que dificultan el proceso de optimización. Por último, se ha demostrado que el proceso calibración validación no evalúa la idoneidad del modelo porque conjuntos diferentes de parámetros pueden ser óptimos, lo que se conoce como el problema de "equifinalidad" (Beven y Ferrer, 2001). El problema de equifinalidad puede generar un comportamiento hidrológico equivocado. Por lo tanto, el conocimiento del sistema a través de análisis de sensibilidad y el juicio experto es una buena alternativa para calibrar modelos hidrológicos. La figura 2 muestra los caudales simulados y observados a escala diaria para el punto de cierre de la cuenca del río Aipe durante el período de calibración.

A pesar de que la inspección visual es una métrica subjetiva del nivel de ajuste del modelo, en la figura 2 se observa como el modelo representa la textura de la señal medida en campo; durante la mitad de la señal la subestima y hacia el final la sobrestima. Sin embargo, se evidencia un ajuste razonable entre la señal observada y simulada durante este período, con un coeficiente de determinación de $\mathrm{R}^{2}=0.75$ (figura 3), muestra que el 75\% de los datos el modelo los predice y el $25 \%$ restante son incertidumbres asociados a la estructura del modelo conceptual, la calidad de la información ambiental, errores en la medición de los caudales, sobrestimación de parámetros del modelo, etc.

Tabla 3. Rango de valores estimados de los parámetros del modelo weap en la cuenca del río Aipe

\begin{tabular}{l|c|c|c|c|c|c|c|c}
\hline \multirow{2}{*}{ Coberturas } & \multirow{2}{*}{$\begin{array}{c}\text { SWC } \\
(\mathbf{m m})\end{array}$} & \multicolumn{2}{c|}{ RRF } & \multicolumn{2}{c|}{ RZC (mm/día) } & \multirow{2}{*}{ Ks } & \multirow{2}{*}{ PFD } & $\begin{array}{c}\text { Z1 }^{*} \\
\text { (\%) }\end{array}$ \\
\hline Aguas abiertas & 290 & 2 & 7.2 & 200 & 150 & 2 & 0.5 & 20 \\
\hline Bosques & 330 & 2 & 9.5 & 230 & 160 & 6 & 0.4 & 20 \\
\hline Café & 260 & 2 & 9.5 & 140 & 100 & 4 & 0.5 & 20 \\
\hline Pastos & 255 & 2 & 7 & 170 & 100 & 3 & 0.6 & 20 \\
\hline Suelos al desnudo & 230 & 2 & 7.2 & 200 & 160 & 3 & 0.6 & 20 \\
\hline Zonas agrícolas & 260 & 2 & 7.2 & 190 & 100 & 4 & 0.5 & 20 \\
\hline Zonas urbanas & 230 & 2 & 7.2 & 200 & 150 & 1 & 0.9 & 20 \\
\hline Arroz & 260 & 2 & 9.5 & 190 & 80 & 4 & 0.6 & 20 \\
\hline Cacao & 260 & 2 & 7.2 & 190 & 80 & 4 & 0.5 & 20 \\
\hline Lagos & 290 & 2 & 7.2 & 200 & 150 & 2 & 0.5 & 20 \\
\hline Sorgo & 260 & 2 & 7.2 & 180 & 80 & 4 & 0.6 & 20 \\
\hline
\end{tabular}

$* \mathrm{DC}=5 \mathrm{~mm} / \mathrm{dí}, \mathrm{DWC}=500 \mathrm{~mm}$ y $\mathrm{Z2}=30 \%$. 


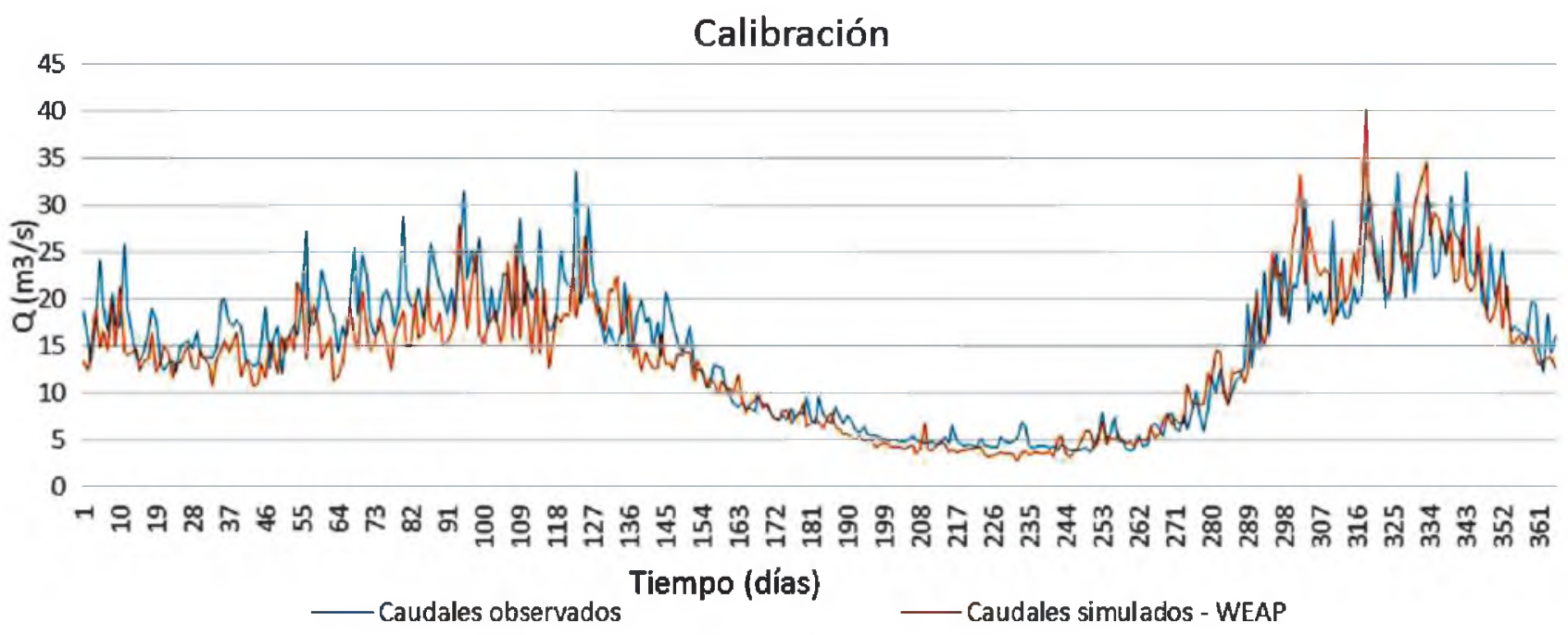

Figura 2. Caudales simulados y observados en el punto de cierre de la cuenca del río Aipe durante período de calibración.

\section{CALIBRACION}

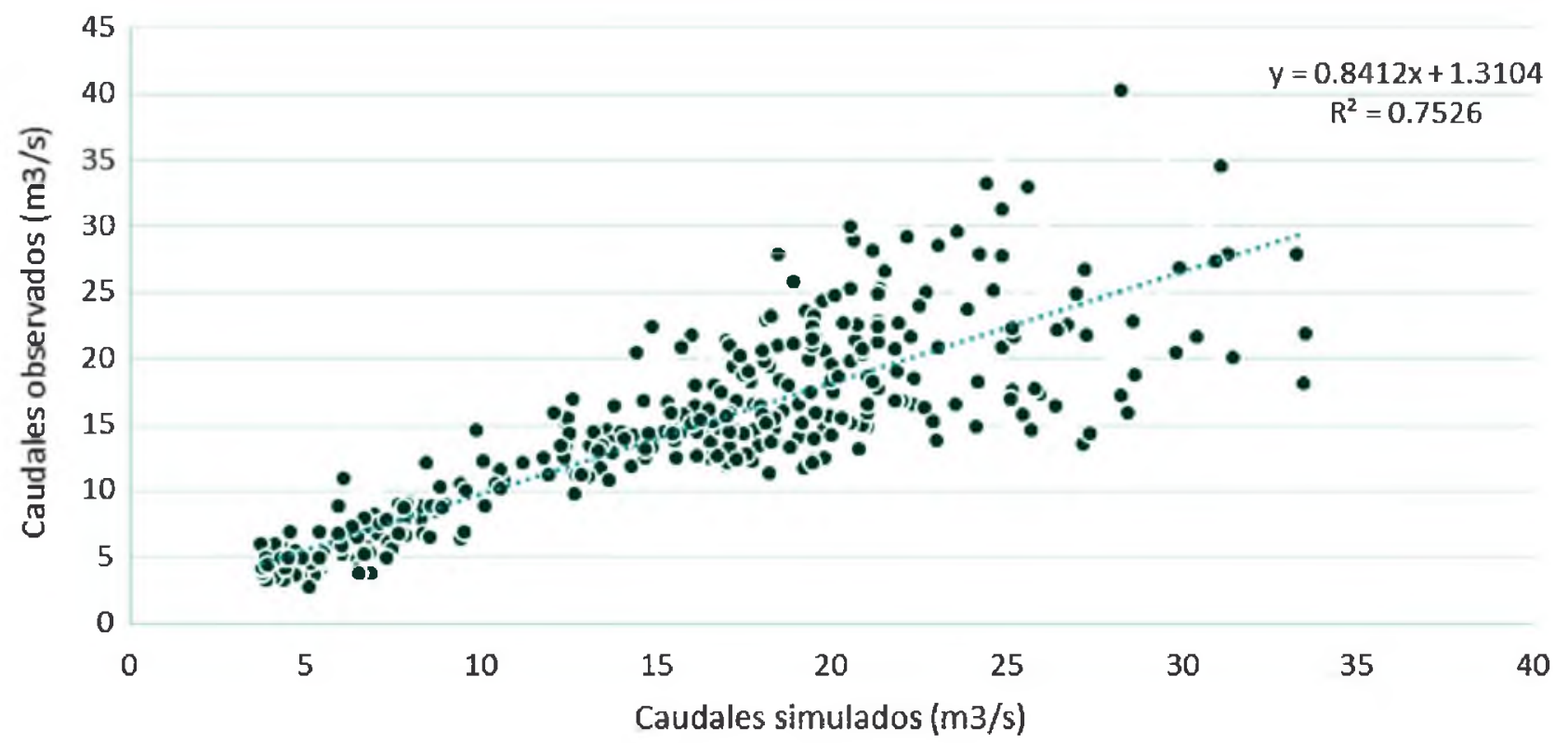

Figura 3. Diagrama de dispersión para los caudales simulados y observados en la cuenca del río Aipe.

\subsection{Validación del modelo}

Para el período de validación el desempeño del modelo fue un poco más bajo $\mathrm{R}^{2}=0.57$. Sin embargo, el modelo sigue manteniendo una buena representación de la variabilidad estacional de la cuenca (figura 4). Cuando los modelos hidrológicos muestran un bajo desempeño en la validación usualmente se infiere la no estacionariedad de los parámetros. En este caso se estaría incumpliendo el supuesto de estacionariedad, el cual puede estar asociado a cambios bruscos por efectos antrópicos.

En la figura 4 se identifica el efecto hidrológico de los dos períodos de lluvia típico de los Andes Colombianos. Para el país existen dos estaciones: verano (temporada de menos lluvia) e invierno (temporada de más lluvia) que están asociadas con el desplazamiento hacia el norte y sur del país de la zona de confluencia intertropical (ZCI) en el año (Henríquez, 2012). El 


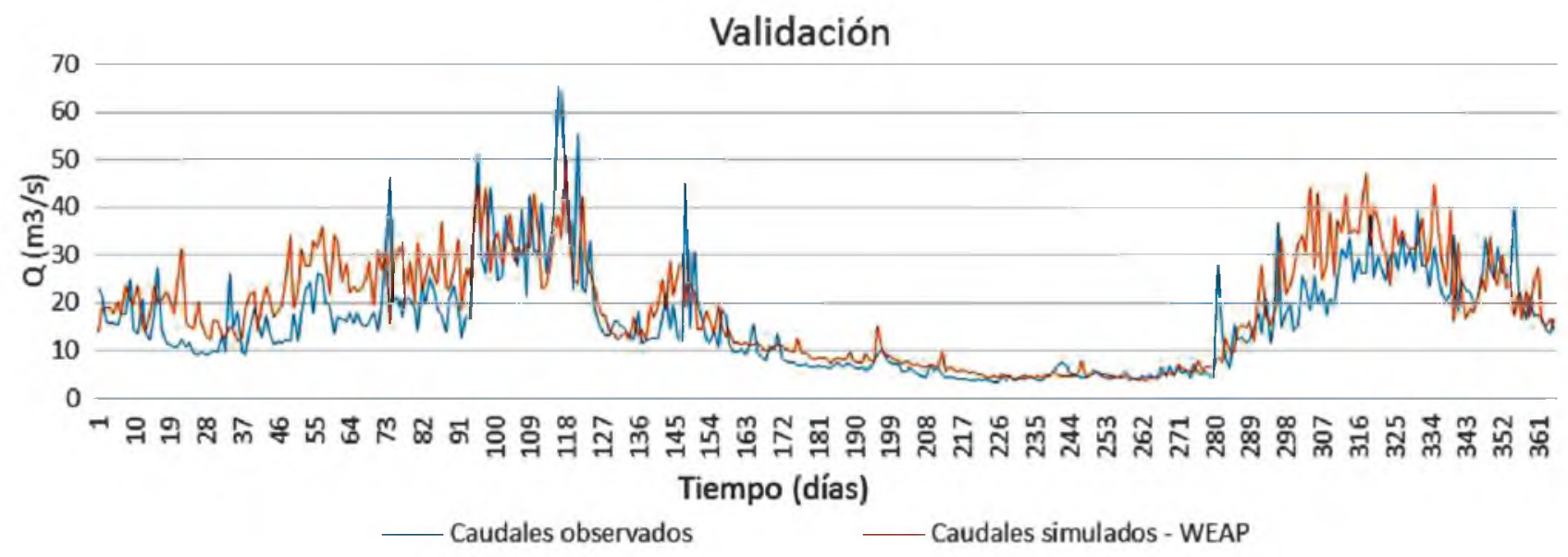

Figura 4. Caudales simulados y observados en el punto de cierre de la cuenca del río Aipe durante período de validación.

modelo representa adecuadamente los dos períodos de caudales máximos. Sin embargo, el modelo tiene la tendencia a sobreestimar el caudal base y no representa exactamente los caudales máximos. A pesar de estos problemas, el modelo reproduce la textura de la señal observada. Estos resultados muestran que el modelo es capaz de representar la dinámica hidrológica de la cuenca del río Aipe a escala diaria. Contrastando estos resultados con los obtenidos por Romero, et al., (2015) donde se obtuvo un $\mathrm{R}^{2}=0.89$ para el período de validación con modelos agregados (Thornthwaite y Thomas) en la cuenca del rio Aipe, se evidencia un mayor ajuste de los modelos agregados frente al modelo weap. Sin embargo, hay que tener en cuenta que los resultados con los modelos agregados son a escala mensual y los del modelo weap a escala diaria, esta diferencia hace que los modelos estén representando procesos hidrológicos diferentes y no necesariamente comparables. Además, pese a que el modelo weap tiene un menor desempeño, es un modelo semi-distribuido que estaría generando más entendimiento de los procesos hidrológicos y produciendo más información a nivel espacial.

\subsection{Escenarios de simulación}

Se simularon cuatro posibles escenarios (tabla 2) con el propósito de analizar el impacto en la respuesta hidrológica de la cuenca del río Aipe. Los cambios que describen los escenarios en términos de crecimiento poblacional y variabilidad climática pueden estar presentes en un futuro no muy lejano. Las simulaciones hidrológicas involucran el proceso de calibración y validación del modelo que se realizó con información históricay del presente para identificar los parámetros que mejor describen la firma hidrológica de la cuenca y de esta forma tener una línea base para comparar la respuesta de los escenarios.

\subsection{Impacto a futuro de incremento de población y cambio climático}

La variable incremento de población es presentada en los escenarios I y II. Estos escenarios asumen que la población del municipio de Aipe continúa su crecimiento y por tanto su demanda de agua potable también incrementa. Este crecimiento no necesariamente tiene que ser por incremento de la tasa de natalidad, también puede obedecer a procesos de migraciones o incremento de población flotante debido a una apuesta de las actividades turísticas. La variación de la oferta hídrica de la cuenca del río Aipe en relación a la línea base (1980 - 2011) para la simulación del escenario I $(+2 \%)$ muestra una disminución de $0.43 \mathrm{~m}^{3} \mathrm{~s}^{-1}$ y para el escenario II $(+10 \%)$ de $4.44 \mathrm{~m}^{3} \mathrm{~s}^{-1}$ (figura 6). De estos resultados se puede deducir que la fuente será suficiente para abastecer de agua potable al municipio de Aipe. Sin embargo, aparte de estos procesos antrópicos, se deben considerar los impactos naturales debidos a eventos de sequías y variaciones de precipitaciones en espacio y tiempo.

Por otro lado, teniendo en cuenta la variable clima se presentan los escenarios III y IV. Para estos escenarios, se muestran dos series de clima MPI-ESM-MR, que hacen referencia a un clima húmedo y seco, los cuales se muestran en la figura 5. Con respecto a los datos históricos de la estación Puente Carretera cuando el clima es húmedo hay un aumento de $3.67 \mathrm{~m}^{3} \mathrm{~s}^{-1}$, lo que indica que el caudal en la cuenca del río Aipe incrementa un 23.97\% para el período (2011-2050). Por el contrario, cuando el clima es seco el caudal 


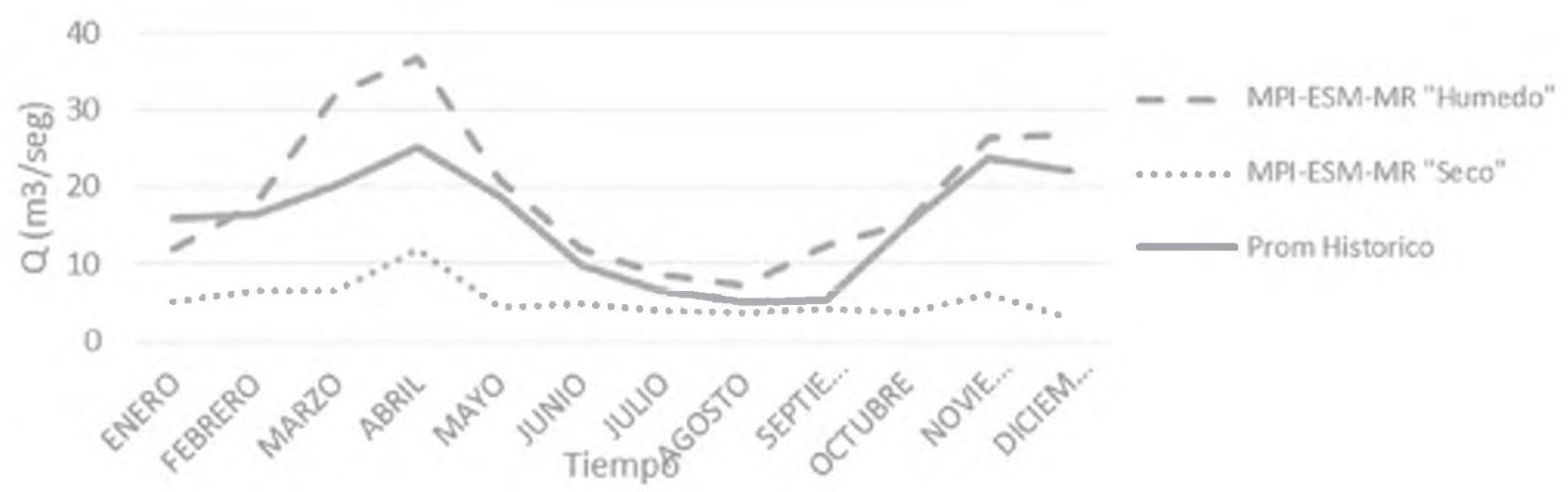

Figura 5. Simulación de caudales con dos escenarios de clima.

disminuye $10.07 \mathrm{~m}^{3} \mathrm{~s}^{-1}$, mostrando una reducción muy marcada del $65.77 \%$ (figura 6 ).

De acuerdo con la figura 5 los escenarios mantienen la estacionalidad de la climatología característica de los Andes Colombianos pero con diferente orden de magnitud. En general se observan dos períodos marcados de lluvias a finales de los meses de abril y noviembre, lo que es característico de los climas tropicales como los de Colombia. Esto se debe a la influencia de los dos océanos que rodean el país, como lo son el océano Pacifico, el Atlántico y la Amazonia (funcionando como un tercer océano). Estos períodos de lluvias también son generados por la cercanía de Colombia con la línea del Ecuador y la zona de convergencia intertropical, donde convergen los vientos alisios del hemisferio Norte con los del hemisferio Sur. Se caracteriza por ser un ancho cinturón de baja presión constituido por corrientes de

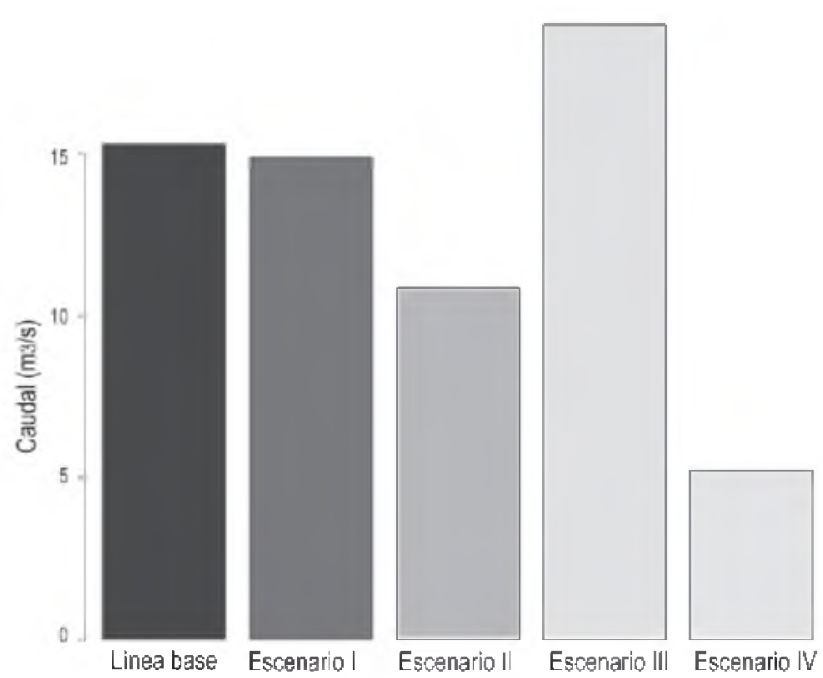

Figura 6. Diagrama de barras que muestra el impacto de los escenarios sobre el caudal en el punto de cierre de la cuenca del río Aipe. aires ascendentes donde convergen grandes masas de aire cálido y húmedo proveniente del norte y del sur de la zona intertropical (León y Eslava, 2000; Henríquez, 2012). Esta variabilidad hidrológica interanual asociado al patrón de las lluvias también fue documentada por Legesse et al., (2003) en la cuenca tropical semiárida del río Ketar en el sur de África. Los escenarios de clima demuestran que la precipitación es una entrada de agua muy importante para la cuenca. En otras palabras y de acuerdo con los resultados de Montenegro y Ragab (2012) la escorrentía de la cuenca es muy sensible a los cambios en el régimen de lluvias. Sin embargo, para el escenario de clima seco (IV) se evidencia que la cuenca es más reactiva a un incremento de las lluvias que a un escenario de estiaje, a tal punto que el escenario IV (más pesimista) estaría proyectando una sequía prolongada donde la cuenca solo estaría funcionando con el flujo base producto del acuífero (figura 5).

La figura 6 compara el impacto de los escenarios sobre el caudal en la estación de cierre de la cuenca. La cual brinda un abanico de posibles respuestas hidrológicas de la cuenca del río Aipe ante un futuro incierto pero que se debe estar preparado para afrontar.

Sin embargo, el peor escenario sería la combinación de alta demanda de agua (escenario III) y poca producción de escorrentía superficial (escenario IV) lo que podría ocasionar numerosos problemas económicos y conflictos sociales. Por ejemplo, que pasaría con la tradicional producción de arroz que utiliza grandes volúmenes de agua de riego por superficie (sistema altamente ineficiente y que promueve el uso irracional del agua), la producción de tilapia en tierra que actualmente tiene tanta demanda a nivel nacional e internacional. En este contexto, es necesario dimensionar medidas de adaptación acordes con los escenarios 
climáticos. Por ejemplo, (i) promoción de cultivos de secano que tengan requerimientos hídricos menos exigentes, (ii) aprovechar especies y semillas nativas que no alteren la dinámica ecohidrológica, (iii) incrementar la capacidad de infiltración y recarga de la cuenca para alimentar el acuífero a través de mayores tiempos de retención hídrica, (iv) promover prácticas de cosecha de agua lluvia, (v) establecer prácticas de diseño hidrológico para retener agua aprovechando la topografía de la cuenca (línea clave). De acuerdo con lo expuesto, el aporte de este trabajo se concentra en poder mejorar el sistema de gestión de recursos hídricos y asistir a la toma de decisiones, a través de conocimiento confiable de cómo responden las cuencas de aporte ante distintos escenarios climáticos y de demandahídrica.

Finalmente, los resultados muestran la habilidad del modelo para predecir el comportamiento hidrológico de la cuenca del río Aipe, cuenca de clima tropical con heterogeneidad y anisotropía en los suelos y la vegetación que introducen una complejidad natural en la firma hidrológica de la cuenca. A pesar de los problemas asociados a la disponibilidad y la calidad de la información ambiental, el modelo mostró un buen desempeño y la representación de la dinámica hidrológica de la cuenca del río Aipe se puede considerar confiable. Respecto a los escenarios, los relacionados con el ensamble de cambio climático (III y IV), es necesario tratar estos resultados con un grado de incertidumbre. De acuerdo con los resultados de Arnell (2011) en cuencas de Inglaterra, Nóbrega, et al., (2011) en el Brasil y Gosling et al., (2011) en cuencas de varias partes del mundo, estos modelos de circulación global tienen un alto grado de incertidumbre asociado al modelo climático (a pesar de ser modelos de base física). En este sentido, sería necesario evaluar la incertidumbre de los modelos climáticos, los escenarios y la propagación de esta incertidumbre en el modelo hidrológico antes de que estos resultados apoyen la toma de decisiones a nivel político (Prudhomme, et al., 2003).

\section{Conclusiones}

El estudio de modelación hidrológica se realizó en una cuenca semiárida de clima tropical en el sur de Colombia para abordar un problema de gestión de recursos hídricos con planificación integral a largo plazo incluyendo escenarios de cambio climático. Se seleccionó el modelo weap para conocer el impacto de los escenarios de cambio climático sobre la oferta hídrica del río Aipe para el período (2011-2050). El modelo fue capaz de simular el comportamiento hidrológico de la cuenca hidrográfica del río Aipe cuando fue comparado el caudal simulado y observado en el punto de cierre de la cuenca (estación puente carretera). En general y a pesar de los problemas asociados a la disponibilidady la calidad de la información ambiental, el modelo mostró un buen desempeño $\left(\mathrm{R}^{2}=0.75\right)$ y la representación de la dinámica hidrológica de la cuenca del río Aipe se puede considerar confiable. Los escenario de demanda hídrica (I y II) y cambio climático (III y IV) fueron simulados con los parámetros identificados en la calibración del modelo. Respecto a la simulación de los escenarios, la línea base (1980-2011) estimó un caudal medio de $15.31 \mathrm{~m}^{3} \mathrm{~s}^{-1}$, el escenario I estimó un caudal de $14.88 \mathrm{~m}^{3} \mathrm{~s}^{-1}(-2.81 \%)$, el escenario II de $10.87 \mathrm{~m}^{3} \mathrm{~s}^{-1}(-29 \%)$, escenario III de 18.98 $\mathrm{m}^{3} \mathrm{~s}^{-1}(+23.97 \%)$ y escenario IV de $5.24 \mathrm{~m}^{3} \mathrm{~s}^{-1}(-$ $65.77 \%$ ). El modelo hidrológico desarrollado para la cuenca del río Aipe representa una herramienta útil que asiste el proceso de planeamiento hidrológico a partir de escenarios multicriterios que tienen en cuenta los múltiples y opuestos usos del recurso hídrico. Finalmente, el modelo calibrado y validado con las condiciones ambientales de la cuenca contribuye a mejorar el sistema de gestión integral del recurso hídrico y apoyar la toma de decisiones, a través de conocimiento confiable de cómo responden las cuencas en términos hidrológico ante distintos escenarios climáticos y de demanda hídrica.

\section{Agradecimientos}

Especial agradecimiento a la Corporación Autónoma Regional del Alto Magdalena (CAM), United States Agency for International Development (USAID), Stockholm Environment Institute (SEI), The Nature Concervancy (Andrés Angarita) y los evaluadores del artículo.

\section{Referencias bibliográficas}

Angarita, H., 2014. Metodología para incluir variabilidad climática y escenarios de cambio climático en el modelo WEAP de la macro cuenca del Magdalena y resultados de las simulaciones. The Nature Conservancy. Bogotá. 47pp.

Arnell, N.W., 2011. Uncertainty in the relationship between climate forcing and hydrological response in UK catchments. Hydrol. Earth Syst. Sci. 15, 897-912. 
Arrojo, P., Del Moral, L., Herrera, T., 2015. El agua: Perspectiva ecosistémica y gestión integrada. Fundación Nueva Cultura del Agua. España. 444pp.

Banos-González, J., Martínez-Fernández, M.A., EsteveSelma, M., 2016. Using dynamic sustainability indicators to assess environmental policy measures in Biosphere Reserves. Ecological Indicators. 67, 565-576.

Baños González, I., Martínez Fernández, J., Esteve Selma, M., 2014. Simulación dinámica de sistemas socio-ecológicos: sostenibilidad en Reservas de la Biosfera. Revista Ecosistemas. 22(3), 74-83.

Becerra, R.A., 2011. Efecto del Cambio Climático en la Evolución de la Cobertura Nival de la Cuenca Alta del Río Maipo. [Trabajo de grado]. Universidad de Chile: Santiago de Chile, Chile.

Beven, K.J., 2001. Rainfall-Runoff Modelling - the Primer, Wiley, Chichester, UK. 356pp.

Beven, K.J., Freer J., 2001. Equifinality, data assimilation, and uncertainty estimation in mechanistic modelling of complex environmental systems using the GLUE methodology. J. Hydrol. 249(1-4), 11-29.

Castro, N. L., 2014. Implementación del sistema de modelación WEAP como herramienta para la gestión integral del recurso hídrico en la vereda $\mathrm{La} \mathrm{Be}$ 1la. [Trabajo de grado]. Universidad Tecnológica de Pereira: Pereira, Colombia.

Duque, L.F., Vázquez, R.F., 2015. Modelación de la Oferta Hídrica en una Cuenca de Montaña Tropical en Función de su Cobertura del Suelo. Water Availability Modelling for a Tropical Mountain Catchment as a Function of its Soil Cover. AquaLAC. 7(1), 63-76.

Francés, F., Vélez, J. I, Vélez, J.J., 2007. Split-parameter structure for the automatic calibration of distributed hydrological models. J. Hydrol. 332, 226-240.

Gosling, S. N., Taylor, R. G., Arnell, N. W., Todd, M. C., 2011. A comparative analysis of projected impacts of climate change on river runoff from global and catchment-scale hydrological models. Hydrol. Earth Syst. Sci. 15, 279-294.

Gupta, V.H., Sorooshian, S., Yapo, P. O., 1998. Toward improved calibration of hydrologic models: Multiple and noncommensurable measures of information. Water Resour. Res. 34(4), 751- 763.

Henríquez, M.D., 2012. Climatología ambiental de Colombia. Una guía sobre la relación clima-ambiente para los estudiantes y profesionales de las carreras ambientales. Universidad Santo Tomás. Facultad de Ingeniería Ambiental. Bogotá D.C. 330pp.

Legesse, D., Vallet-Coulomb, C., Gasse, F., 2003. Hydrological response of catchment to climate and land use changes in Tropical Africa: case study South Central Ethiopia. J. Hydrology. 275, 67-85.

Lema, M.A., Plaza, V.C., 2009. Modelación hidrológica de la cuenca (alta y media) del río Pastaza aplicando el modelo de simulación WEAP (Water Evaluation and Plannig System). [Trabajo de grado]. Universidad de Chile: Santiago de Chile, Chile.

Leon G., Zea, J., Eslava, J., 2000. Circulación general del trópico y la zona de confluencia intertropical en Colombia. Meteorología Colombiana. 1, 31-38.

Martínez-Fernández, J., Esteve-Selma, M.A., BañosGonzález, I., Carreño, F., Moreno, A., 2013. Sustainability of Mediterranean irrigated agrolandscapes. Ecological Modelling. 248, 11-19.

Martínez, J., Esteve, M.A., 2004. Dynamics of water scarcity on irrigated landscapes: Mazarron and Aguilas in Southeastern Spain. System Dynamics Review. 20 (2), 117-137.

Mardones, G.A., 2009. Implementación y Uso del Modelo Weap en Cuencas Nivales de la IV Región para el Análisis del Cambio Climático. [Trabajo de grado]. Universidad de Chile: Santiago de Chile, Chile.

Mena, D., 2009. Análisis del Cambio Climático en la Cuenca Andina del Río Teno, Usando el Modelo WEAP. [Trabajo de grado]. Universidad de Chile: Santiago de Chile, Chile.

Montenegro, S., Ragab, R., 2012. Impact of possible climate and land use changes in the semi arid regions: A case study from North Eastern Brazil. J. Hydrology. 434-435, 55-68.

Nóbrega, M. T., Collischonn, W., Tucci, C. E. M., Paz, A. R., 2011. Uncertainty in climate change impacts 
on water resources in the Rio Grande Basin, Brazil, Hydrol. Earth Syst. Sci. 15, 585-595.

Pardo, D.I., 2009. Análisis de Impactos del Cambio Climático en la Cuenca Andina del Río Teno, Usando el Modelo Weap. [Trabajo de grado]. Universidad de Chile: Santiago de Chile, Chile.

Pineda, J.N., Resendiz, C.P., 2014. Aplicación del Modelo de Simulación Water Evaluation and Planning System (WEAP), para la gestión integral de la cuenca del Río Fuerte. [Trabajo de grado]. Instituto Politécnico Nacional, México.

Prudhomme, C., Jakoba, D., Svenssona, C., 2003. Uncertainty and climate change impacto $n$ the flood regime of small UK catchments. J. Hydrol. 277, 1-23.

Romero, J., Buitrago, A., Quintero, T., 2014. Evaluación del Efecto de los Escenarios del Cambio Climático Sobre el Recurso Hídrico en las Cuencas del Rio Yaguara y Aipe. Universidad Surcolombiana (USCO): Neiva, Huila.

Ruiz-Villanueva, V., Stoffel, M., Bussi, G., Francés, F., Bréthaut, C., 2015 Climate changes impacts on discharges of the Rhone River in Lyon by the end of the twenty-first century: model results and implications. Regional Environmental Change. 15 (3), 505-515.

Ruiz, J.F., 2010. Cambio climático en temperatura, precipitación y humedad relativa para Colombia usando modelos meteorológicos de alta resolución. Panorama 2011 - 2100. Nota técnica 005/2010 del IDEAM.
Ruiz-Pérez, G., Medici, C., Latron, J., Llorens, P., Gallart, F., Francés, F., 2016 Investigating the behaviour of a small Mediterranean catchment using three different hydrological models as hypotheses. Hydrol. Process., doi: 10.1002/hyp.10738.

Salinas, A.A., Paz, O., 2011. Aplicación del Modelo de Planificación Hídrica de Cuencas Weap al Proyecto: Aducción de Recursos Hídricos Mururata. 6. Revista Tecnología, Investigación y Docencia. $6,27-38$.

Sieber, J., Purkey, D., 2007. WEAP. Water Evaluation and Planning System USER GUIDE for WEAP21. Stockholm Environment Institute. US Center, 219pp.

Sivapalan, M., Savenije, H. H. G., y Blöschl, G., 2012. Socio-hydrology: A new science of people and water. Hydrol. Process. 26, 1270-1276.

Suárez, J., Puertas, J., Anta, J., Jácome, A., ÁlvarezCampana, J.M., 2014. Gestión integrada de los recursos hídricos en el sistema del agua urbana: Desarrollo Urbano Sensible al Agua como enfoque estratégico Ingeniería de Agua. 18, 111-123.

Thompson, J.R., Green, A.J., Kingston, D.G., Gosling, S.N., 2013. Assessment of uncertainty in river flow projections for the Mekong River using multiple GCMs and hydrological models, Journal of Hydrology. 486, 1-30.

Xue, L., Yue, Z., Chunli S., Jian S., Zhong-Liang, W., Yuqiu W., 2015. Application of Water Evaluation and Planning (WEAP) model for water resources management strategy estimation in coastal Binhai New Area, China. Ocean \& Coastal Management. 106, 97-109. 
